\title{
A Mild Method for the Formation and in situ Reaction of Imidoyl Chlorides: Conversion of Pyridine-1-oxides to 2-Aminopyridine
} Amides

\author{
Peter J. Manley and Mark T. Bilodeau* \\ Department of Medicinal Chemistry, Merck Research Laboratories, P. O. Box 4, West Point, PA 19486.
}

\section{Supplementary Material}

General experimental information: Proton and carbon NMR spectra were recorded on Varian Unity 400 or VRX-400 spectrometers (400 MHz) or Varian Unity or Varian Plus $(500 \mathrm{MHz})$ spectrometers. Chemical shifts are reported in part per million (d) downfield from tetramethylsilane as an internal standard. High resolution mass spectra were recorded on a Bruker 3T Fourier transform ion cyclotron resonance mass spectrometer equipped with electrospray ionization. The $\mathrm{MH}+$ signal of the analyte of interest is mass measured against the internal calibrant, polypropylene glycol (average mass (425). Solvents and reagents were obtained from commercial sources and used without further purification. The reported yields are the actual isolated yields of purified material and are not optimized.

General procedure: To a solution of 1.5 equivalents of secondary amide in $\mathrm{CH}_{2} \mathrm{Cl}_{2}(0.2$ $\mathrm{M}$ with respect to pyridine-1-oxide) was added 2.5 equivalents of 2,6-lutidine then 1.5 equivalents of oxalyl chloride dropwise at $0^{\circ} \mathrm{C}$ resulting in the evolution of gas. After stirring for 15 minutes 1 equivalent of pyridine-1-oxide was added all at once and the cooling bath was removed. After the N-oxide had been consumed as judged by TLC or LC/MS the mixture was diluted with saturated $\mathrm{NaHCO}_{3}$ and extracted with $\mathrm{CH}_{2} \mathrm{Cl}_{2}(3 \mathrm{x})$. The combined organic layers were dried $\left(\mathrm{MgSO}_{4}\right)$, filtered, and concentrated. The residue was purified by flash column chromatography using $\mathrm{MeOH} / \mathrm{CH}_{2} \mathrm{Cl}_{2}$ or EtOAc/hexanes solvent mixtures as indicated.

$N$-methyl- $N$-pyridin-2-ylacetamide (Table 1, entry 1): Purification using 0-5\% $\mathrm{MeOH} / \mathrm{CH}_{2} \mathrm{Cl}_{2}$ gradient gave a yellow oil $(58 \mathrm{mg}, 73 \%)$ : ${ }^{1} \mathrm{H}-\mathrm{NMR}(500 \mathrm{MHz}, \mathrm{CDCb}) \delta$ 8.50 (d, $1 \mathrm{H}, \mathrm{J}=4.64 \mathrm{~Hz}), 7.76$ (m, $1 \mathrm{H}), 7.30$ (bs, $1 \mathrm{H}), 7.20$ (t, $1 \mathrm{H}, \mathrm{J}=7.08 \mathrm{~Hz}$ ), 3.39 (s, $3 \mathrm{H}), 2.10$ (s, $\left.3 \mathrm{H}) ;{ }^{13} \mathrm{C}-\mathrm{NMR}(100 \mathrm{MHz}, \mathrm{CDC}\}\right) \delta 171.0,149.1,138.4,122.0,120.7$, 35.7, 23.3; HRMS calc for $\mathrm{C}_{8} \mathrm{H}_{11} \mathrm{~N}_{2} \mathrm{O}$ 151.0866, found 151.0874 .

$N$-benzyl- $N$-pyridin-2-ylacetamide (Table 1, entry 2): Purification using 0-5\% $\mathrm{MeOH} / \mathrm{CH}_{2} \mathrm{Cl}_{2}$ gradient gave a mixture. A second purification using $30 \%$ EtOAc hexanes gave a pale yellow oil $(94 \mathrm{mg}, 79 \%):{ }^{1} \mathrm{H}-\mathrm{NMR}\left(500 \mathrm{MHz}, \mathrm{CDCb}_{3}\right) \delta 8.50(\mathrm{~d}, 1$ $\mathrm{H}, \mathrm{J}=4.64 \mathrm{~Hz}), 7.67$ (m, $1 \mathrm{H}), 7.28-7.06$ (m, $7 \mathrm{H}), 5.11$ (s, $2 \mathrm{H}), 2.08$ (s, $3 \mathrm{H}) ;{ }^{13} \mathrm{C}-\mathrm{NMR}$ 
$\left(100 \mathrm{MHz}, \mathrm{CDCb}_{3}\right) \delta 149.4,138.3,137.8,128.7,128.1,127.4,122.3,121.9,51.3,23.4$; HRMS calc for $\mathrm{C}_{14} \mathrm{H}_{15} \mathrm{~N}_{2} \mathrm{O} 227.1179$, found 227.1172.

$N$-phenyl- $N$-pyridin-2-ylacetamide (Table 1, entry 3): Purification using 25\% EtOAc hexanes gave a yellow oil $(63 \mathrm{mg}, 56 \%):{ }^{1} \mathrm{H}-\mathrm{NMR}\left(500 \mathrm{MHz}, \mathrm{CDCl}_{3}\right) \delta 8.43(\mathrm{~m}, 1 \mathrm{H})$, $7.72(\mathrm{~m}, 1 \mathrm{H}), 7.48-7.25(\mathrm{~m}, 6 \mathrm{H}), 7.13(\mathrm{~m}, 1 \mathrm{H}), 2.11(\mathrm{~s}, 3 \mathrm{H}) ;{ }^{13} \mathrm{C}-\mathrm{NMR}(100 \mathrm{MHz}$, $\left.\mathrm{CDCl}_{3}\right) \delta$ 171.2, 155.5, 149.0, 142.3, 138.1, 129.7, 128.6, 127.8, 121.7, 121.4, 24.5; HRMS calc for $\mathrm{C}_{13} \mathrm{H}_{13} \mathrm{~N}_{2} \mathrm{O} 213.1023$, found 213.1030.

$N$-methyl- $N$-pyridin-2-ylbenzamide (Table 1, entry 4): Purification using 0-5\% $\mathrm{MeOH} / \mathrm{CH}_{2} \mathrm{Cl}_{2}$ gradient gave a mixture. A second purification using $25 \%$ EtOAc hexanes gave a clear oil (112 mg, 100\%): ${ }^{1} \mathrm{H}-\mathrm{NMR}(500 \mathrm{MHz}, \mathrm{CDC}$ ) $) \delta 8.45(\mathrm{~m}, 1 \mathrm{H})$, $7.43(\mathrm{~m}, 1 \mathrm{H}), 7.35-7.21(\mathrm{~m}, 5 \mathrm{H}), 7.04(\mathrm{~m}, 1 \mathrm{H}), 6.81(\mathrm{~d}, 1 \mathrm{H}, \mathrm{J}=8.06 \mathrm{~Hz}), 3.58(\mathrm{~s}, 3 \mathrm{H})$; ${ }^{13} \mathrm{C}-\mathrm{NMR}\left(100 \mathrm{MHz}, \mathrm{CDCl}_{3}\right) \delta 171.2,157.0,148.9,137.5,136.3,130.3,128.7,128.2$, 121.8, 121.1, 36.2; HRMS calc for $\mathrm{C}_{13} \mathrm{H}_{13} \mathrm{~N}_{2} \mathrm{O} 213.1023$, found 213.1023.

$N$-benzyl- $N$-(4-phenylpyridin-2-yl)acetamide (Table 2, entry 1): Purification using 95:5 $\mathrm{DCM} / \mathrm{MeOH}$ and then using 70:30 hexanes/EtOAc gave a colorless oil $(151 \mathrm{mg}, 86 \%)$ : ${ }^{1} \mathrm{H}-\mathrm{NMR}(500 \mathrm{MHz}, \mathrm{CDCl}) \delta 8.53(\mathrm{~d}, 1 \mathrm{H}, \mathrm{J}=5.1 \mathrm{~Hz}), 7.52-7.44(\mathrm{~m}, 6 \mathrm{H}), 7.39(\mathrm{dd}, 1 \mathrm{H}$, $\mathrm{J}=1.5,5.4 \mathrm{~Hz}), 7.30-7.21(\mathrm{~m}, 5 \mathrm{H}), 5.15\left(\mathrm{~s}, 2 \mathrm{H0}, 2.12(\mathrm{~s}, 3 \mathrm{H}) ;{ }^{13} \mathrm{C}-\mathrm{NMR}(100 \mathrm{MHz}\right.$, $\left.\mathrm{CDCl}_{3}\right) \delta 170.8,156.1,149.7,137.9,129.7,129.4,128.7,128.2,127.5,127.2,120.4$, 119.8, 51.4, 23.5; HRMS calc for $\mathrm{C}_{20} \mathrm{H}_{18} \mathrm{~N}_{2} \mathrm{O} 303.1492$, found 303.1467.

$N$-[4-(benzyloxy)pyridin-2-yl]- $N$-methylacetamide (Table 2, entry 2): Purification using 95:5 DCM/MeOH gave a colorless oil (91 mg, 67\%): ${ }^{1} \mathrm{H}-\mathrm{NMR}\left(500 \mathrm{MHz}, \mathrm{CDCl}_{3}\right) \delta$ $8.31(\mathrm{~d}, 1 \mathrm{H}, \mathrm{J}=5.6 \mathrm{~Hz}), 7.43-7.35(\mathrm{~m}, 5 \mathrm{H}), 6.85(\mathrm{bs}, 1 \mathrm{H}), 6.81(\mathrm{dd}, 1 \mathrm{H}, \mathrm{J}=2,2,5.8 \mathrm{~Hz})$, $5.14(\mathrm{~s}, 2 \mathrm{H}), 3.34(\mathrm{~s}, 3 \mathrm{H}) ;{ }^{13} \mathrm{C}-\mathrm{NMR}(100 \mathrm{MHz}, \mathrm{CDCl}) \delta 170.9,166.6,158.0,150.0$, 135.5, 129.0, 128.7, 127.71, 127.68, 109.4, 107.4, 70.4, 35.7, 23.2; HRMS calc for $\mathrm{C}_{15} \mathrm{H}_{16} \mathrm{~N}_{2} \mathrm{O}_{2} 257,1285$, found 257.1277.

$\mathrm{N}$-methyl- $\mathrm{N}$-(4-methylpyridin-2-yl)acetamide (Table 2, entry 3): Purification using $90 \%$ EtOAc hexanes gave a yellow oil $(62 \mathrm{mg}, 61 \%)$ : ${ }^{1} \mathrm{H}-\mathrm{NMR}(500 \mathrm{MHz}, \mathrm{CDCb}) \delta 8.34(\mathrm{~d}$, $1 \mathrm{H}, \mathrm{J}=5.13 \mathrm{~Hz}), 7.08$ (bs, $1 \mathrm{H}), 7.03(\mathrm{~d}, 1 \mathrm{H}, \mathrm{J}=4.64 \mathrm{~Hz}), 3.35(\mathrm{~s}, 3 \mathrm{H}), 2.40(\mathrm{~s}, 3 \mathrm{H})$, 2.07 (s, $3 \mathrm{H}) ;{ }^{13} \mathrm{C}-\mathrm{NMR}\left(100 \mathrm{MHz}, \mathrm{CDCl}_{3}\right) \delta 170.9,150.0,148.8,123.2,121.4,35.7$, 23.2, 21.2; HRMS calc for $\mathrm{C}_{9} \mathrm{H}_{12} \mathrm{~N}_{2} \mathrm{O}$ 164.0950, found 164.0948.

$N$-methyl- $N$-(3-methylpyridin-2-yl)acetamide (Table 2, entry 4, major): Purification using $80 \%$ EtOAc hexanes gave a yellow solid (30 mg, 40\%): ${ }^{1} \mathrm{H}-\mathrm{NMR}(500 \mathrm{MHz}$, $\left.\mathrm{CDCl}_{3}\right) \delta 8.41(\mathrm{~d}, 1 \mathrm{H}, \mathrm{J}=3.42 \mathrm{~Hz}), 7.67(\mathrm{~d}, 1 \mathrm{H}, \mathrm{J}=7.33 \mathrm{~Hz}), 7.26(\mathrm{~m}, 1 \mathrm{H}), 3.22(\mathrm{~s}, 3$ H), $2.29(\mathrm{~s}, 3 \mathrm{H}), 1.80(\mathrm{~s}, 3 \mathrm{H}) ;{ }^{13} \mathrm{C}-\mathrm{NMR}\left(100 \mathrm{MHz}, \mathrm{CDCl}_{3}\right) \delta$ 170.5, 155.5, 147.7, 140.6, 130.6, 123.9, 34.4, 22.2, 17.3; HRMS calc for $\mathrm{C}_{9} \mathrm{H}_{12} \mathrm{~N}_{2} \mathrm{O}$ 164.0950, found 164.0953 .

$N$-methyl- $N$-(5-methylpyridin-2-yl)acetamide (Table 2, entry 4, minor): Purification using $80 \%$ EtOAc hexanes gave a yellow oil $(18 \mathrm{mg}, 24 \%):{ }^{1} \mathrm{H}-\mathrm{NMR}(500 \mathrm{MHz}, \mathrm{CDCl}$ ) $\delta 8.32(\mathrm{~s}, 1 \mathrm{H}), 7.56(\mathrm{~d}, 1 \mathrm{H}, \mathrm{J}=7.81 \mathrm{~Hz}), 7.14(\mathrm{bs}, 1 \mathrm{H}), 3.34(\mathrm{~s}, 3 \mathrm{H}), 2.36(\mathrm{~s}, 3 \mathrm{H}), 2.04$ 
(s, $3 \mathrm{H}) ;{ }^{13} \mathrm{C}-\mathrm{NMR}\left(100 \mathrm{MHz}, \mathrm{CDCl}_{3}\right) \delta 170.9,149.4,139.0,120.3,35.7,23.1,18.1$; HRMS calc for $\mathrm{C}_{9} \mathrm{H}_{12} \mathrm{~N}_{2} \mathrm{O}$ 164.0950, found 164.0948.

$N$-benzyl- $N$-(6-methylpyridin-2-yl)acetamide (Table 2, entry 5): Purification using 30\% EtOAc in hexanes gave a colorless oil $(290 \mathrm{mg}, 74 \%):{ }^{1} \mathrm{H}-\mathrm{NMR}(500 \mathrm{MHz}, \mathrm{CDCb}) \delta$ $7.52(\mathrm{t}, 1 \mathrm{H}, \mathrm{J}=7.8 \mathrm{~Hz}), 7.27(\mathrm{~m}, 5 \mathrm{H}), 7.3(\mathrm{~d}, 1 \mathrm{H}, \mathrm{J}=7.6 \mathrm{~Hz}), 6.80(\mathrm{bs}, 1 \mathrm{H}), 5.08(\mathrm{~s}, 2 \mathrm{H})$, $2.54(\mathrm{~s}, 3 \mathrm{H}), 2.05(\mathrm{~s}, 3 \mathrm{H}) ;{ }^{13} \mathrm{C}-\mathrm{NMR}(100 \mathrm{MHz}, \mathrm{CDCl}) \delta 170.6,158.8,154.8,138.4$, 138.0, 128.8, 128.6, 128.3, 127.3, 121.9, 118.9, 51.3, 24.5, 23.3; HRMS calc for $\mathrm{C}_{15} \mathrm{H}_{16} \mathrm{~N}_{2} \mathrm{O} 241.1335$, found 241.1333.

$N$-methyl- $N$-quinolin-2-ylacetamide (Table 2, entry 6): Purification using 30\% EtOAc hexanes gave a pale yellow solid (50 mg, 73\%): ${ }^{1} \mathrm{H}$-NMR $\left(500 \mathrm{MHz}, \mathrm{CDCb}_{3}\right) \delta 8.20(\mathrm{~d}$, $1 \mathrm{H}, \mathrm{J}=8.54 \mathrm{~Hz}), 7.99(\mathrm{~d}, 1 \mathrm{H}, \mathrm{J}=8.54 \mathrm{~Hz}), 7.83(\mathrm{~d}, 1 \mathrm{H}, \mathrm{J}=8.05 \mathrm{~Hz}), 7.74(\mathrm{t}, 1 \mathrm{H}, \mathrm{J}=$ $7.57 \mathrm{~Hz}), 7.56(\mathrm{t}, 1 \mathrm{H}, 7.81 \mathrm{~Hz}), 7.46(\mathrm{bs}, 1 \mathrm{H}), 3.51(\mathrm{~s}, 3 \mathrm{H}), 2.21(\mathrm{~s}, 3 \mathrm{H}) ;{ }^{13} \mathrm{C}-\mathrm{NMR}$ $\left(100 \mathrm{MHz}, \mathrm{CDCl}_{3}\right) \delta 171.3,155.4,147.1,138.4,130.3,128.9,127.6,126.8,126.7,119.0$, 35.6, 23.8; HRMS calc for $\mathrm{C}_{12} \mathrm{H}_{13} \mathrm{~N}_{2} \mathrm{O} 201.1023$, found 201.1031.

$N$-isoquinolin-1-yl- $N$-methylacetamide (Table 2, entry 7): Purification using 0-5\% $\mathrm{MeOH} / \mathrm{CH}_{2} \mathrm{Cl}_{2}$ gradient gave a brown oil $(62 \mathrm{mg}, 90 \%):{ }^{1} \mathrm{H}-\mathrm{NMR}(500 \mathrm{MHz}, \mathrm{CDCl}) \delta$ $8.46(\mathrm{~d}, 1 \mathrm{H}, \mathrm{J}=5.62 \mathrm{~Hz}), 7.95$ (m, $2 \mathrm{H}$ ), 7.79-7.67 (m, $3 \mathrm{H}), 3.41$ (s, $3 \mathrm{H}), 1.79$ (s, $3 \mathrm{H})$; ${ }^{13} \mathrm{C}-\mathrm{NMR}\left(100 \mathrm{MHz}, \mathrm{CDCl}_{3}\right) \delta 171.1,155.5,142.1,138.7,131.3,128.9,127.6,125.3$, 124.6, 121.7, 35.8, 22.7; HRMS calc for $\mathrm{C}_{12} \mathrm{H}_{13} \mathrm{~N}_{2} \mathrm{O}$ 201.1023, found 201.1033. 